Classification

Physics Abstracts

66.10

\title{
LONG TAILS IN THE TIME CORRELATION FUNCTIONS OF A TWO-DIMENSIONAL SYSTEM OF DIATOMIC MOLECULES
}

\author{
C. TRESSER, B. QUENTREC $\left({ }^{*}\right)$ and C. BROT \\ Laboratoire de Physique de la Matière Condensée $\left({ }^{* *}\right)$ \\ Parc Valrose, 06034 Nice, France
}

(Reçu le 25 avril 1977, accepté le 31 mai 1977)

\begin{abstract}
Résumé. - Une simulation numérique (Dynamique Moléculaire) nous a permis d'étudier le comportement aux temps longs de certaines fonctions d'autocorrélation pour un système bidimensionnel de 1600 molécules diatomiques à densité moyenne. Nous avons bien observé la décroissance théorique en $1 / t$ pour l'autocorrélation de la vitesse linéaire. Pour la vitesse angulaire nous observons un comportement inattendu en $1 / t^{3}$.
\end{abstract}

Abstract. - By a molecular dynamics simulation of $4 \times 10^{4}$ steps we study the long-time behaviour of the autocorrelation functions of a bidimensional system of 1600 diatomic molecules at intermediate density. The predicted $1 / t$ behaviour for the velocity autocorrelation is observed. A surprising $1 / t^{3}$ behaviour is observed for the angular velocity autocorrelation.

Since the discovery of the non exponential behaviour of the linear velocity autocorrelation function by Alder and Wainwright [1], there has been much interest in this problem [2] and if we do not take into account the two-dimensional divergence problem the situation is reasonably clear concerning the asymptotic behaviour for spherical molecules : the velocity ACF has a long time tail decaying as $t^{-d / 2}$, where $d$ is the dimension of the system.

But the situation is not so clear concerning the rotational autocorrelation functions of a non-spherical molecule. Phenomenological approaches (hydrodynamic) as well as mode-mode coupling or kinetic ones couple the individual rotational behaviour of the molecule with long lived modes. They all lead to a $t^{-5 / 2}$ decay for a three dimensional system [ $3 a$ to $3 e$ ]. The hydrodynamic approach generalized to a $d$ dimensional system predicts a $t^{-(d+2) / 2}$ decay [3f].

As far as we know at the time of writing, no experimental results are available. The aim of this letter is to give the results of a molecular dynamics experiment on this problem.

We take a two-dimensional system of 1600 diatomic molecules with the usual periodic boundary conditions. The interaction potential is an atom-atom potential as used for instance in the simulation of liquid nitro-

(*) Present address : Chemistry Department, Massachusetts Institute of Technology, Cambridge, Massachusetts 02139, USA.

(**) Laboratoire associé au C.N.R.S. no 190. gen $[4,5]$. The potential between two atoms of two different molecules is

$$
\begin{array}{ll}
v(r)=4 \varepsilon(\sigma / r)^{12}-(\sigma / r)^{6}+\varepsilon & \text { for } r \leqslant 2^{1 / 6} \sigma \\
v(r)=0 & \text { for } r>2^{1 / 6} \sigma
\end{array}
$$

where $r$ is the distance between the two atoms. We shall take $\varepsilon$ and $\sigma$ as units of energy and length, respectively. Adding the mass of the molecule, all the physical quantities of interest can be given with these units.

The half distance between the two atoms of the molecule $d=0.3 \sigma$. This is the largest value which does not give a dumbbell shape to the molecule, in the sense defined in reference [4]. We choose a rather elongated molecule in the hope that the rotational coupling of the individual molecule with the collective modes will be important. The temperature of the system is $T=1.2 \varepsilon / k_{\mathrm{B}}$, the density $p=0.17$ molecule $/ \sigma^{2}$. This number density gives a surface density equal to 0.32 . This is of the same order as that used by Alder and Wainwright for one of their hard disks experiments [6] and may be compared with the closepacking density of equivalent ellipses, which is 0.907 .

We performed four runs of $10^{4}$ steps each; the two last steps of a run provided the initial conditions for the following one. We used the usual algorithm of Verlet [7]. Our step was 0.01 in the reduced time unit : $\sigma \sqrt{m / \varepsilon}$. In all the $4 \times 10^{4}$ steps we never saw a numerical fluctuation of the total energy of more than 
$1 / 1000$. The search for the neighbours was performed by a method proposed by two of us some time ago [8]. For a large number of molecules this method gives an important gain in time compared to the usual one [7]. We used the IBM 370-168 of C.I.R.C.E. with double precision. Each run lasted about an hour. The dynamical variables of interest were recorded on tape after every 9 steps. The time interval for the calculation of the correlation functions is $H=0.18 \sigma \sqrt{m / \varepsilon}$. An important problem in obtaining the asymptotic behaviour is to avoid the echo due to periodic boundary conditions. Since we had no information on the sound velocity in our model system, we performed a simulation of 5000 steps on one hundred molecules with the same conditions of density and temperature as in the long runs. The time of appearence of artificial oscillations in the autocorrelation functions computed from this test enabled the sound velocity in the system to be estimated. We found $c=0.75 \sigma / H$. Hence with 1600 molecules the echo should appear for times greater than $130 \mathrm{H}$. Our correlation functions are computed up to $100 \mathrm{H}$. So we are sure that interference phenomena due to the echo do not affect our results.

We have computed the following autocorrelation functions :

$$
\begin{aligned}
& F_{v}(t)=\langle\mathbf{v}(0) \mathbf{v}(t)\rangle\left\langle\left\langle\mathbf{v}^{2}\right\rangle\right. \\
& F_{j}(t)=\langle\mathbf{j}(0) \mathbf{j}(t)\rangle /\left\langle\mathbf{j}^{2}\right\rangle \\
& F_{u}(t)=\langle\mathbf{u}(0) \mathbf{u}(t)\rangle
\end{aligned}
$$

where $\mathbf{v}, \mathbf{j}$ and $\mathbf{u}$ are respectively the linear velocity, the angular velocity and the unit orientational vector of one molecule.

The asymptotic behaviour being very small, it is of crucial interest to have a good estimate of the numerical error [10]. Comparing the results of the four independent runs, we found that the discrepancy between two runs was never more than 0.007 for $F_{v}(t)$ and $F_{u}(t)$ and never more than 0.009 for $F_{j}(t)$. So, an estimate of the bounds for the absolute numerical error for $10^{4}$ steps is \pm 0.004 for $F_{v}(t)$ and $F_{u}(t)$ and \pm 0.005 for $F_{j}(t)$. The absolute error for $F_{j}(t)$ is greater than for $F_{v}(t)$ and $F_{u}(t)$ because, for a two dimensional system, $\mathbf{v}$ and $\mathbf{u}$ have two components while the angular velocity $\mathbf{j}$ has only one. Averaging the results for the four independent runs, we get an estimate of the numerical error which is \pm 0.002 for $F_{v}(t)$ and $F_{u}(t)$ and \pm 0.003 for $F_{j}(t)$.

In figure $1, F_{v}(t), F_{j}(t)$ and $F_{u}(t)$ are given in a semilogarithmic scale. After a short inertial behaviour, each of the three functions exhibits a first exponential decay $\mathrm{e}^{-t / \tau}$. For $F_{v}(t), \tau_{v}=4.6 \mathrm{H}$, for $F_{j}(t), \tau_{j}=6 \mathrm{H}$ and for $F_{u}(t), \tau_{u}=2.2 \mathrm{H}$.

After times of the order of $10 H$ for $F_{v}, 20 H$ for $F_{j}$ and $10 H$ for $F_{u}$, the ACF's adopt a slower decay. Little is known theoretically about this intermediate regime [2]. On figure 1 , it is seen that these decays can be plotted along several segments of exponen-

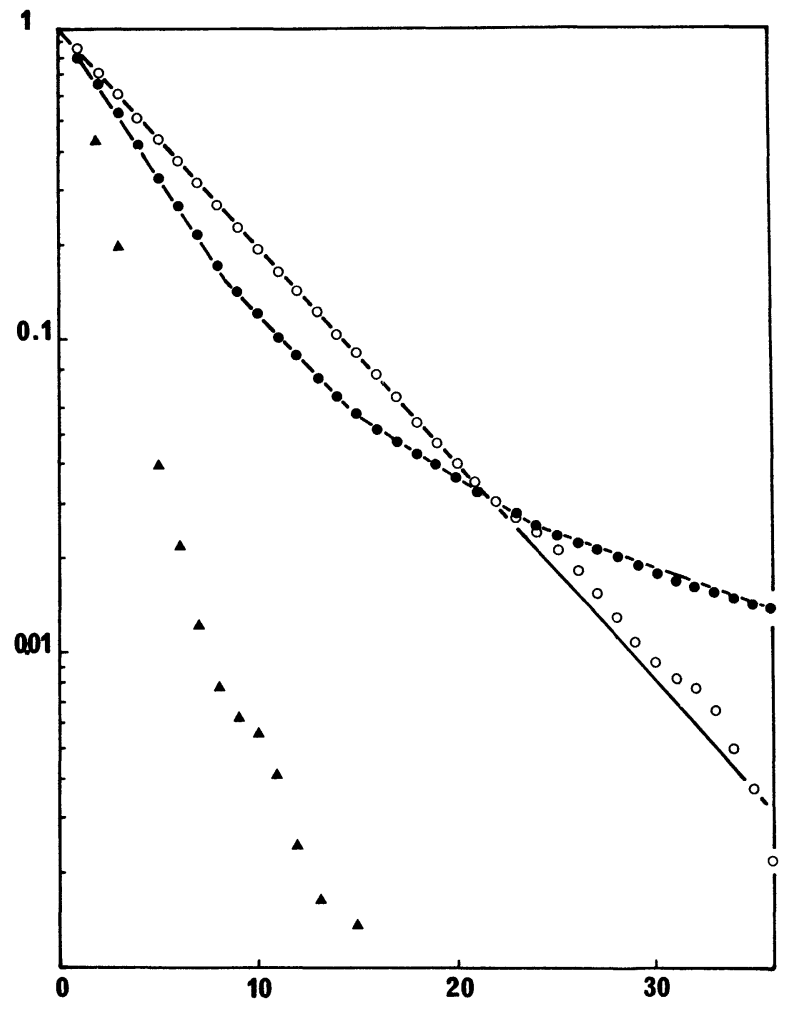

FIG. 1. - Semi-logarithmic plot (decimal logarithm) of the autocorrelation functions. $\mathbf{\Delta} \mathbf{\Delta}: F_{u}(t), \infty: F_{v}(t), \infty 0: F_{j}(t)$. The time is given in $H$ units.

tials $\left(^{1}\right)$. We have no comments to offer on the meaning, or even on the reality, of this behaviour. In fact, in this region the results coud be fitted using algebraic forms as well, with higher exponents than the ones that describe the true asymptotic behaviours (see Fig. 2).

Figure 2 shows $F_{v}(t)$ in a $\ln -\ln$ plot for values of $t$ between $10 \mathrm{H}$ and $100 \mathrm{H}$. For $t$ greater than about $25 H$ up to $100 H$, we observe the expected $1 / t$ behaviour over a very long span of time, about fifteen times the molecular time $\tau_{v}$. Notice that the observed life of this long tail is one and a half times it takes to appear, whereas in reference [9] it is only $70 \%$. This observation confirms the theoretical prediction [2] that the exponent of the algebraic asymptotic behaviour is independent of the shape of the molecule. With our statistics, it is not possible to check the existence of a logarithmic factor as predicted by recent theories $[11,12]\left({ }^{2}\right)$.

Figure 3 shows $F_{j}(t)$ plotted with two time scales : in the insert a $t^{-2}$ time scale, in conformity with the present theoretical predictions, and a $t^{-3}$ scale. It is seen that our results are not linear in the $t^{-2}$ scale. On the other hand, for $t$ between $20 \mathrm{H}$ and $80 \mathrm{H}$, $F_{j}(t)$ fits remarkably a straight line corresponding to

( $\left.{ }^{1}\right)$ The same is true if one plots the velocity AFC given in table I of reference [9].

$\left({ }^{2}\right)$ Yuan, H. and Oppenheim, I., private communication. 


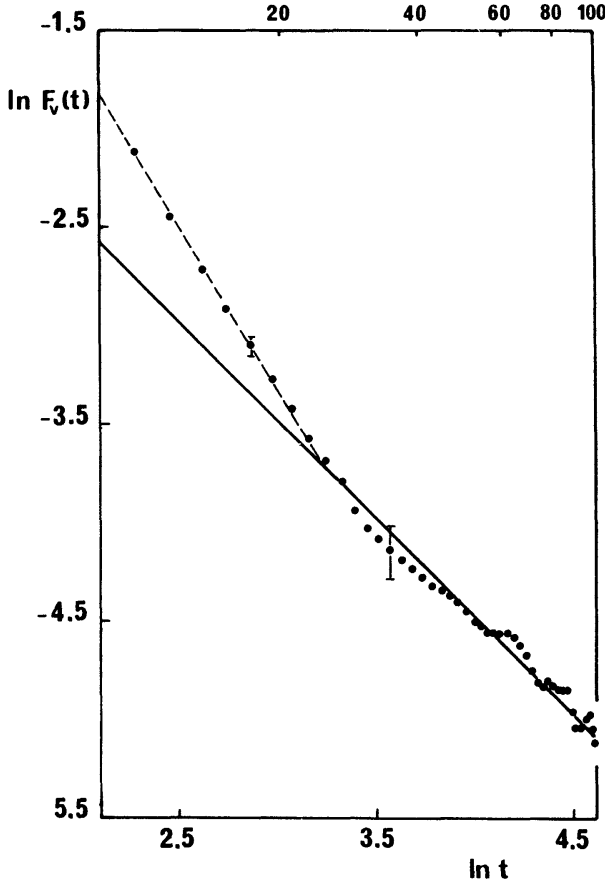

Fig. 2. - Ln-ln plot of $F_{v}(t)$ (neperian logarithm). $\bullet$ denote experimental points. The solid line is a straight line with a slope of -1 . The dashed line is a straight line with a slope of -1.7 . The time is given in $H$ units.

a $t^{-3}-b$ behaviour with $b=0.003$. We note that $b$ is smaller than the statistical error; in another system studied by us, with a different potential, we also observe a long time $t^{-3}$ behaviour, but with $b$ closer to zero (to be published). We do not wish to

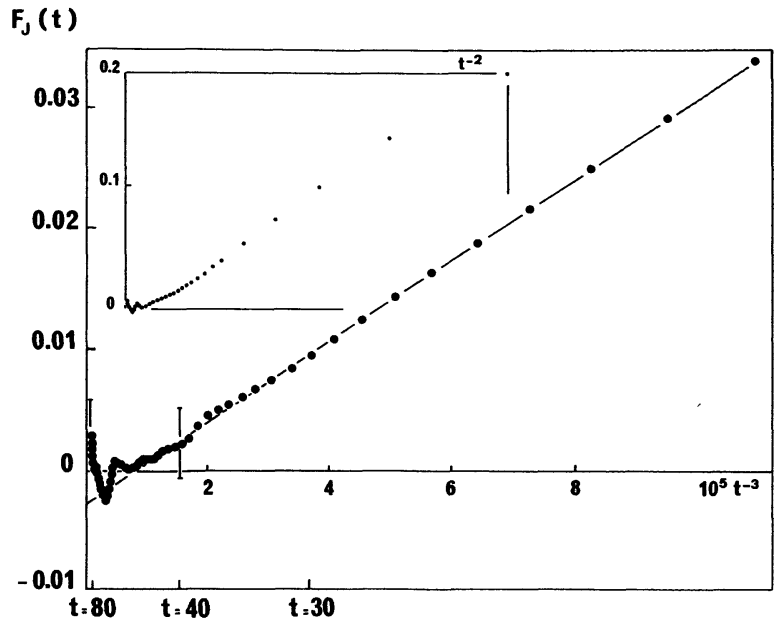

Fig. 3. - Plot of $F_{j}(t)$ as a function of $1 / t^{3}$. $\bullet$ denote experimental points. The last point on the left corresponds to $t=100$. The time is given in $H$ units. In the insert is shown the same function plotted with $t^{-2}$ abscissa.

argue at length here to try to decide whether this very long $t^{-3}$ behaviour is really asymptotic or not. It is probably not; the true long time tail in $t^{-2}$ as most theories predict, would then appear at still longer times and be of very small amplitude [13]. If the $t^{-3}$ behaviour is really asymptotic then the phenomenological basis of the current theories should be revised [14].

Finally, we note that $F_{u}(t)$ decays too fast, at the adopted density, for a numerical test of the controversial possible long tail to be feasible.

\section{References}

[1] Alder, B. J. and Wainwright, T. E., Phys. Rev. Lett. 18 (1967) 968.

[2] For a review see :

Pomeau, Y. and Resibois, P., Phys. Lett. 19c (1975) 63.

[3a] BERNE, B. J., J.C.P. 56 (1972) 2164.

[3b] Ailawadi, N. K. and Harris, S., J. Chem. Phys. 56 (1972) 5783.

[3c] Garisto, F. and Kapral, R., Phys. Rev. A 10 (1974) 309.

[3d] Keyes, T. and Oppenheim, I., Physica 75 (1974) 583.

[3e] Pomeau, Y. and Weber, J., Phys. Rev. A 8 (1973) 1422.

[3f] Allawadi, N. K. and Berne, B. J., J. Chem. Phys. 54 (1971) 3569.

[4] Quentrec, B. and Brot, C., Phys. Rev. A 12 (1975) 272.

[5] Cheung, P. S. Y. and Powles, J. P., Mol. Phys. 30 (1975) 921.
[6] Alder, B. J. and Wainwright, T. E., Phys. Rev. A 1 (1970) 18.

[7] Verlet, L., Phys. Rev. 165 (1968) 201.

[8] QuentreC, B. and Brot, C., Journal of Computational Physics 13 (1973) 430.

[9] Levesque, D. and Ashurst, W. T., Phys. Rev. Lett. 33 (1974) 277.

[10] Zwanzig, R. and Ailawadi, N., Phys. Rev. 182 (1969) 280

[11] Alder, B., Wainwright, T. and Gass, D., Phys. Rev. A 4 (1971) 233.

[12] Forster, D., Nelson, D. R. and Stephen, M. J., Phys. Rev. Lett. 36 (1976) 867.

[13] Mehaffey, J. R., Desai, R. C. and Kapral, R., J. Chem. Phys. 66 (1977) 1665

[14] Evans, D. J., Mol. Phys. 32 (1976) 1171. 